\title{
Computer-Aided Problem Solving - Part 2: A Dialogue-Based System to Support the Analysis of Inventive Problems
}

\author{
Niccolò Becattini ${ }^{1}$, Yuri Borgianni ${ }^{2}$, Gaetano Cascini ${ }^{1}$, and Federico Rotini ${ }^{2}$ \\ ${ }^{1}$ Politecnico di Milano, Dip. di Meccanica, Via Giuseppe la Masa, 1, 20156 Milan, Italy \\ ${ }^{2}$ Università di Firenze, Dip. di Mecc. e Tecn. Ind., Via Santa Marta, 3, 50139 Florence, Italy \\ Niccolo.Becattini@kaemart.it \\ Gaetano.Cascini@polimi.it \\ \{Yuri.Borgianni, Federico.Rotini\}@unifi.it
}

\begin{abstract}
The paper illustrates an original model and a dialogue-based software application that have been developed by integrating the logic of ARIZ with some OTSM-TRIZ models, in order to guide a user, also with no TRIZ background, to the analysis of inventive problems. The dialogue-based procedure brings to the construction of a model of the inventive problem, which is used both to trigger new solutions by highlighting different solving perspectives and to start an automatic knowledge search within technical and scientific information. The prototype system has been tested with students at Politecnico di Milano and at the University of Florence. The paper details the structure of the algorithm and the results of the first validation activity.
\end{abstract}

Keywords: Computer-Aided Innovation, problem solving, OTSM-TRIZ, conceptual design, dialogue-based system.

\section{Introduction}

Despite TRIZ is recognized among its expert practitioners as a theory which efficiently improves individuals and organizations problem solving capabilities, its wide dissemination is still dramatically limited by the learning efforts required to master its tools and to assimilate its original thinking logic. Among the main causes which hinder its diffusion in the industrial world, a relevant role is certainly played by the investment needed to introduce TRIZ logic and tools in the existing product development cycle. Several organizational and educational models have been proposed so far, as also in [1], but still several critical open issues remain:

- The percentage of people who starts adopting some TRIZ instruments after attending an introductory course is very limited.

- "Simplified TRIZ", too often intended as a fuzzy application of the contradiction matrix and the inventive principles, is closer to a brainstorming session with guided "stimuli" rather than to the TRIZ problem solving process, and indeed its potential is much more limited. Thus, a conflict exists between a proper assimilation of a TRIZ "way of thinking" and the time available in the industrial reality to learn and practice. 
- Such a conflict is even tougher for SMEs, since in a small organization each employee covers several roles and it is quite impossible to dedicate sufficient time and efforts to TRIZ learning, while keeping the other functions.

- Several TRIZ-based software applications have been proposed in the market since the '90s, but these systems are not useful to speed up the TRIZ learning process and are marginally usable by people without any TRIZ background. Indeed, many companies, that in the past acquired some licenses of these applications without a proper TRIZ education, largely contribute to the promotion of a bad image of TRIZ.

Especially in the last decade, the literature witnesses the need to create TRIZ-based tools addressed to help non-practitioners, with the double purpose of offering intuitive ready-to-use problem solving skills and providing a learning approach to fully take advantage of TRIZ capabilities. In such perspective Dubois et al. [2] propose an algorithmic framework to support the representation of contradictions and standard solutions. In the same context, the authors decided to start a research activity aimed at defining a new role for TRIZ-based computer applications, i.e. CAI problem-solving "coaches" for non-trained users. According to the authors' intention, being guided by a computer application, a designer with no TRIZ background should be able to improve his problem solving capability since the first usage of the software and at the same time to acquire the ARIZ logic through a learn-by-doing process.

The overall motivation of the research is further detailed in [3], where the general requirements of a computerized system for problem solving are identified through a detailed discussion about typical classifications of engineering problems, approaches to problem solving and related computer implementations, the advantages of a dialogue-based human-machine interaction to elicit his knowledge and stimulate his creative skills.

On the basis of the insights arising from this survey, the authors hereby propose an original dialogue-based system, founded on TRIZ logic, and suitable for software implementation.

According to the conclusions drawn in [3], here assumed as a starting point for the development of the computer-implementable algorithm, in order to support inventive problem solving activities within the conceptual design stage, CAI systems should overcome the dichotomy between cognitive and systematic problem solving models, both to exploit user's knowledge and to guide the design activity towards the solution with a step by step process. The system should also allow an abstract description of the problem in order to enlarge the solutions space and to link the design process with relevant external sources of information from different expertise domains. Eventually, the adoption of a natural language dialogue-based interaction with the user can be considered as an effective means to support designers with no specific background on problem solving methodologies, but also to improve their capabilities through a learning-by-doing process.

The paper hence starts with a survey of the conceptual models adopted as a reference to build the computer-implementable problem solving algorithm. Then the structure of the algorithm is detailed, with a careful description of all its modules and an exemplary excerpt from one of them. The second part of the paper describes the experimental activity run with the MS degree and $\mathrm{PhD}$ students in mechanical 
engineering at Politecnico di Milano and University of Florence; the following discussion allows to point out some positive conclusions about the potentialities of the proposed algorithm and also to identify further directions of investigation.

\section{Development of a System to Support the Analysis of Inventive Problems}

The double goal of fully exploiting TRIZ capabilities and envisaging both cognitive and systematic aspects of problem solving methods, as a need emerged by the review reported in [3], has been the basis for the selection of the theoretical pillars and the models to build a Computer-Aided problem solving framework.

Moreover, the mentioned survey has addressed the authors towards the development of a dialogue-based system for assisting the inventive tasks of the conceptual design phase. This section briefly mentions the theoretical reference items and details the original dialogue-based algorithm developed by the authors as the foundation for the software problem solving application.

\subsection{OTSM-TRIZ Models as a Meta-Cognition Framework for Inventive Problem Solving}

As discussed in [3], a synthesis beyond the dichotomy between cognitive and systematic approaches to problem solving allows to avoid trial and error, build efficient procedures, leverage the available knowledge resources of individuals and teams and highlight knowledge lacks to be covered with new information sources.

According to the authors' experience, the most comprehensive and organic suite of models describing a problem solving process with the abovementioned characteristics is provided by OTSM-TRIZ [4] and includes:

- Hill model (abstraction-synthesis);

- Tongs model (from current situation to ideality, barriers identification);

- Funnel model (convergent process);

- System Operator (system thinking).

These models should not be considered as alternative paths for transforming a problematic situation into a solution, but as complementary descriptions of the characteristics of an efficient problem solving process.

As recalled in the Introduction, TRIZ has been proved to be a very useful help to designers for developing innovative products. Its set of tools and concepts allows to provide reliable results when addressing non-typical problems. However, substantial limitations arise due to the considerable learning efforts required to master its logic and tools, as for example witnessed by well known industrial players within the TRIZ community $[5,6]$.

For this reason, a specific goal of the present research is to allow even users without a strong vocational experience to achieve viable conceptual solutions. Moreover, due to the given market boundaries, the recourse to time-consuming and potentially expensive specialization courses has to be excluded. This issue is especially relevant for SMEs, for which a considerable growth in the need to employ 
systems and software for innovation is expected [7], that therefore could be considered the primary users of the tool under development.

Since the innovation system has to be addressed mainly to inexperienced practitioners, particular attention has to be paid, beyond the foolproof use, towards the removal of TRIZ specific terminology. Thus the application has to foresee TRIZ models, but the user/system interface has to be built through a common language, using at the greatest extent terms and concepts introduced by the designer himself/herself.

\subsection{Description of the Algorithm: Logical Blocks and Further Outputs}

The original contribution of this paper is constituted by an algorithm for problem analysis, structured in the form of a dynamic dialogue, suitable for implementation in a software application. The underpinning logic of OTSM-TRIZ and several classical TRIZ tools are integrated in order to widely describe the topic of the investigation and to remark the most relevant issues to be considered for the problem solving activity and, if necessary, for the knowledge search.

The dialogue based system helps at first the user in exploiting his know how by suggesting problem solving paths, that don't require external expertise to be implemented. Thanks to the investigation of the parameters affecting the undesired issues arising in the system, the designer can individuate factors to be modified in order to reformulate the problem as a typical case. Moreover, among the outcomes of the innovation system, the algorithm provides indications for suitable problem solving alternatives through different TRIZ tools, e.g. separating in time/space, trimming useless or low-valued components, opportunities to turn the undesired effect into a useful output, re-thinking the ways to perform the main function or to deliver the same benefits.

However, as already summarized in the introduction, the formalization of the problem should also aid the knowledge search, being the information retrieval within the expertise domain preferable to that in external fields, due to an easier and quicker implementation of the generated ideas. In any case, the knowledge search outside the expertise domain should be accomplished just when the abstraction process has been completely and successfully performed, i.e., when a so called physical contradiction has been correctly formulated.

In order to fulfil the requirements and to cover all the options for problem solving and knowledge search, the framework of the algorithm includes a set of logical blocks aimed at examining different aspects of the system to be designed and/or improved. The network of links among the blocks and the single nodes of the algorithm determine an extensive bundle of paths and cycles to refine the problem formulation, depicted in Fig. 1.

With the objective of easing the usability of the system and the formalization of the problem, the following measures have been taken:

- the nodes of the algorithm are either open questions, choices or messages, intended to provide proper hints in performing the problem solving process;

- the questionnaire employs a common terminology, avoiding TRIZ jargon;

- the text of questions and suggestions resorts to previously introduced terms and items; 


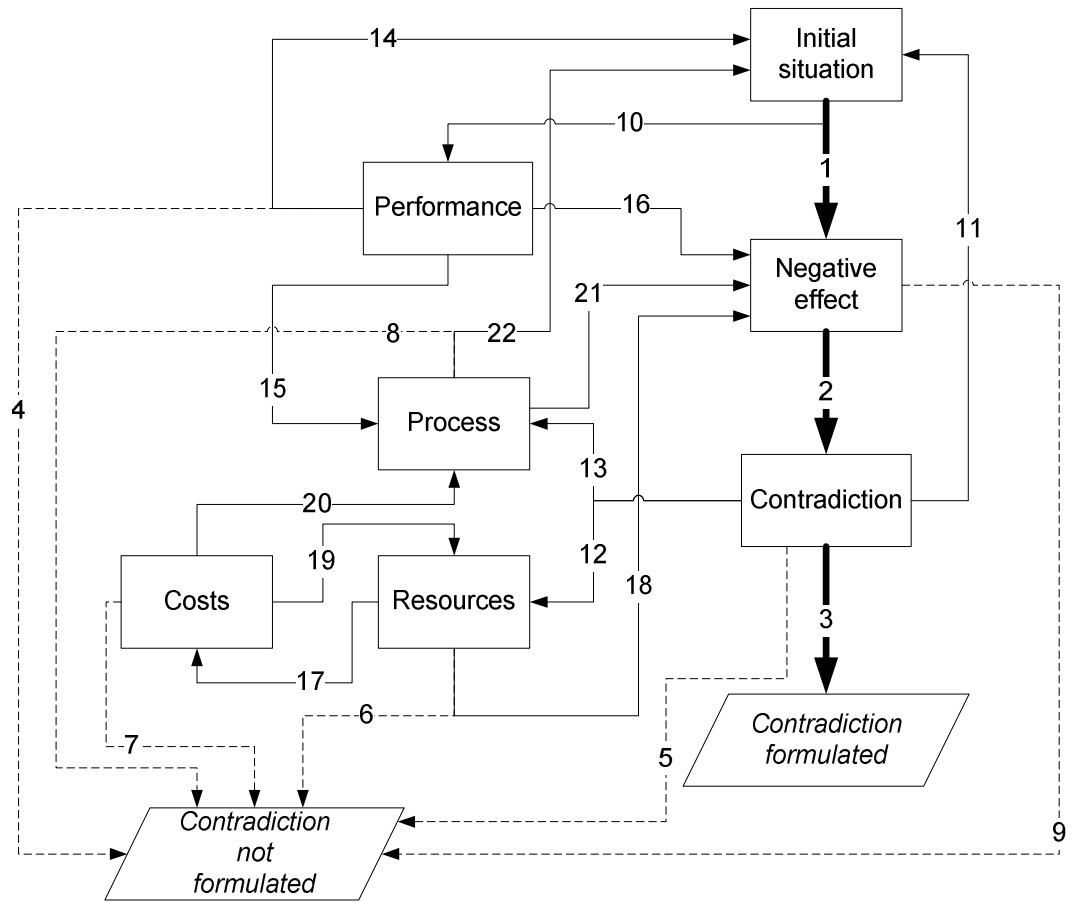

Fig. 1. Network of logical blocks and outputs of the questioning procedure

- some answer examples are provided, as well as their grammatical form, in order to clarify the purpose of the open questions and to provide a more sound text in the downstream nodes of the algorithm;

- the questioning procedure is rich of checks in order to verify the correctness of the user's inputs and to provide a feedback to the user about the outcome of the dialogue.

With the objective of addressing the user towards the most proper description of the situation, the algorithm performs a preliminary distinction among tasks concerning the presence of negative effects or drawbacks, the required implementation of new useful functions and the enhancement of systems with relevant under-performances. The individuation of an undesired effect should lead to the investigation of the features and the phenomena that provoke it and, subsequently, to the formalization of a physical contradiction, grounded on a control parameter leading to mismatching outputs according to the value it assumes. However, even when the user doesn't address the formulation of the problem through the task concerning the elimination of a harmful output, several attempts are carried out with the aim of redefining the model of the system under investigation. The objective is to lead the user towards the formalization of the negative effect responsible for the unsatisfactory or missing functions and, eventually, towards the identification of a contradiction. The most straightforward path for formulating the contradiction, 
remarked in Figure 1 with thicker lines numbered 1, 2 and 3, involves the accomplishment of three logical blocks, intended to assess the initial situation (labelled as 1S), entailing the description of the system through the specification of a wide set of features and correlations with the aim of overcoming psychological inertia, by highlighting also more latent aspects, to define the arising undesired effect (NE) and to identify the conflicting requirements (AR).

However, further ways are foreseen to depict the problem, since several matters can hinder a thorough description of the system under investigation; moreover, lacks of knowledge might occur even within the field of expertise. In case of any circumstance hindering the definition of a contradiction, the algorithm is designed to investigate a wide set of features viable to constitute the core of elements and terms to suggest solution paths or to be sought in proper knowledge bases. The wide description, together with the reformulating attempts, is aided by other logical blocks of the questionnaire, inquiring the designer in order to analyze the circumstances that determine missing functions or cause underperformances (PE), to pinpoint the resources needed by the system to work correctly (RE), to focus on the reasons that imply high costs (CO), to investigate further problems arising during the manufacturing of products or the delivering of services (PR). Eventually, the cases that determine the absence of a contradiction in TRIZ terms, highlighted in Figure 1 with dot lines, include the following:

- the user hasn't seized any possibility to modify the studied system, the phenomena that provoke certain underperformances, the perception of the missed objectives (line 4);

- the attempts to identify a parameter entailing conflicting requirements have failed (line 5);

- the user hasn't succeeded to individuate a proper characterization of the undesired effect in terms of required resources (line 6), high costs (line 7) or problems having reference to any stage of the system lifecycle, whose features are influenced by the design and manufacturing/delivering process (line 8); such characterization is viable, within the questioning procedure, to deepen the description of the system and consequently to reformulate the problem;

- certain individuated criticalities are not considered worth to be further analyzed (line 9).

As explained above, especially in such cases, addressing the problem solving path and knowledge search within the industrial domain of the system to be designed is advised.

Further details about the logical blocks and their connections are provided in the following subsections, within the space limitations of the present paper. Interested readers can get the whole algorithm and even test it, by contacting the authors.

\subsubsection{The Logical Block Initial Situation (IS Block)}

The questioning procedure starts with this block, whereas a preliminary description of the initial situation is performed. The block is aimed at defining, at first, the technical system to be analyzed, its overall goal (meant as the motivation of its existence, the achievements and the outcomes of its use in terms of the desired modifications of a certain state/condition in the world [8]) and the main function it performs. The 
beneficiary of the system and the object subjected to the main function of the system are clarified. The designer is then asked to characterize the technical device under investigation following the hierarchical logic of the System Operator and thus delineating the most relevant components, the proper environment to perform the function, the operative conditions. The user, in order to thoroughly describe the initial situation, is required to delimitate the operative space and zone which are involved when the function is delivered. Subsequently the choice of determining the most proper task for the problem under investigation is carried out. If the designer acknowledges missing functions or relevant underperformances, he/she is addressed towards the block Performance (line 10), otherwise he/she is redirected to the block Negative Effect (line 1). In order to to provide an excerpt of the whole dialogue based procedure, Table 1 illustrates the starting part of the algorithm, including the text of the questions submitted to the users. The Table clarifies whether the single nodes require an open text answer (Assignment - ASS) or the choice between two different options (Decision - DEC); the nodes that follow in each case are also indicated. The last column of the Table summarizes the variables that are introduced by the user and that are subsequently reused in the following questions, where they appear in the square brackets.

\subsubsection{The Logical Block Negative Effect (NE Block)}

The block aims at investigating the undesired effect that arises in the system, as well as the negative consequences and impact. The user is required to indicate, besides the previous items, which element hinder the achievement of the Most Desirable Result by determining the appearance of the negative effect (Tongs model), the operative space and time of such harmful function, alike in ARIZ steps 2.1 and 2.2. A further check is carried out in order to verify whether the element responsible for the undesired effect can be removed from the system without any particular consequence. The accomplishment of the NE block leads the user towards the set of questions that are meant to check the existence of contradiction (AR block, line 2).

\subsubsection{The Logical Block Contradiction (AR Block)}

The block is supposed to allow the definition of a physical contradiction in TRIZ terms. This step represents the final point of the abstraction process, depicted by the top of the Hill model. The user is requested to focus on the parameters, concerning the previously identified element, that influences the extent of the negative effect. The consequences of modifying the parameters, in the sense of reducing the impact of the negative effect, are evaluated up to revealing the decrease of a desired output. The positive effect which is impaired by a modification of the chosen parameter, as well as its operative time and space, are properly defined along the logical block. The mismatching behaviours faced as a result of increasing/decreasing the chosen control parameter constitute the core formulation of the physical contradiction. The knowledge search holds therefore the purpose, analogously to ARIZ step 3.1, to individuate the opportunities of introducing an X-element, capable of removing the negative effect and providing the jeopardized benefits at the maximum extent, like figured out by the Ideal Final Result.

If any parameter is individuated, whose variation (increase or decrease) provides just benefits without any drawback, the procedure suggests to perform such 
modification and to reformulate the problem, thus restarting from the initial IS block (line 11). In case of the impossibility to identify a control parameter leading to the physical contradiction, the algorithm guides the user towards a set of suggestion and a meta-block that supports a further characterization of the undesired effect, addressing subsequently the user towards a deeper investigation, carried out in the RE (line 12) or PR (line 13) blocks.

Table 1. Excerpt of nodes and links belonging to the IS block

\begin{tabular}{|c|c|c|c|c|c|c|}
\hline Step & Text & Type & $\underset{\rightarrow}{\operatorname{Next}}$ & Yes $\rightarrow$ & $\mathrm{No} \rightarrow$ & Variables \\
\hline 1 & $\begin{array}{l}\text { Type the name of the system that is under investigation. } \\
\text { (Use a substantive, without article, i.e. coffee machine, } \\
\text { drill, car...) }\end{array}$ & Start & 2 & & & [SYS] \\
\hline 2 & $\begin{array}{l}\text { Does the problem you want to face involve the whole } \\
\text { [SYS]? }\end{array}$ & DEC & & 4 & 3 & \\
\hline 5 & $\begin{array}{l}\text { Which technical function is carried out by the [SYS] in } \\
\text { order to [GOAL]? } \\
\text { Use the infinitive form of the verb (i.e. keep ink, dry the } \\
\text { clothes, deliver a box...) }\end{array}$ & ASS & 6 & & & [GPF] \\
\hline 12 & $\begin{array}{l}\text { Type who or what perceives the benefits generated by the } \\
\text { [SYS], when it works in order to [GOAL]. }\end{array}$ & ASS & 13 & & & [BEN] \\
\hline 13 & $\begin{array}{l}\text { Describe what or who undergoes the modifications } \\
\text { carried out through the action "to [GPF]". }\end{array}$ & ASS & 14 & & & [OBJ] \\
\hline 16 & $\begin{array}{l}\text { Define the instant or the initial condition in which the } \\
\text { [SYS] starts/start to [GPF]. }\end{array}$ & ASS & 17 & & & [FOTB] \\
\hline 17 & $\begin{array}{l}\text { Define the instant or the end condition in which the } \\
\text { [SYS] stops/stop to [GPF]. }\end{array}$ & ASS & 18 & & & [FOTE] \\
\hline 24 & $\begin{array}{l}\text { Is your task related to an inadequate/unsatisfactory } \\
\text { fulfilment of a desired benefit produced by the [SYS]? }\end{array}$ & DEC & & $\begin{array}{c}\mathrm{PE} \\
\text { block }\end{array}$ & $\begin{array}{c}\mathrm{NE} \\
\text { block }\end{array}$ & \\
\hline
\end{tabular}

\subsubsection{The Logical Block Performance (PE Block)}

The block Performance is addressed to reformulate the system under investigation or the undesired effect. It is accessed whenever the user recognizes any kind of underperformance of the system or the need for introducing a new function. First, it is required to define a performance to be enhanced or satisfied by the implementation of the new function and to explain the motivations for the increase of the performance itself. The user is then asked to individuate who or what would perceive the benefits of the improvements, who or what doesn't allow the enhancements in the current technical system. If any of the previously identified items is viable to be modified, the user is suggested to change the technical system under investigation, working on such object and consequently coming back to IS block (line 14). If the designer individuates that a modification of the production process should be carried out in order to achieve the improvements, the algorithm directs the problem solving procedure towards the PR block (line 15). As a last option the designer is asked to formulate the negative effect of the system in terms of an unsatisfactory performance and consequently to follow the NE block (line 16). 


\subsubsection{The Logical Block Resources (RE Block)}

In several cases, the excessive amount of resources spent by a technical system is considered just as an administrative drawback due to the fulfilment of certain product/service requirement. The block is accessed when the user, failing to formulate the contradiction, assesses that relevant problems arise during the employment of the system. This logical block aims at investigating which resources the system needs for working correctly, classifying them in terms of space, time, information, material and energy. In case the designer judges the direct costs as the most critical resources spent during the system lifecycle, the algorithm guides him/her towards the $\mathrm{CO}$ block (line 17) for analyzing the reasons of the high expenditures. Among the amounts of resources spent the user is asked to determine which ones represent the most challenging criticalities and whether this issue can be assumed as the negative effect to be treated by the algorithm. In case of an affirmative answer, the user is redirected towards the NE block (line 18).

\subsubsection{The Logical Block Costs (CO Block)}

In TRIZ terms, costs are seen as quantities to be prevented having reference to the employment of resources or to inaccuracy of any product development stage. The logical block is aimed at classifying what provokes high costs for the system use, production or maintenance. The resources provoking high costs, as a consequence of their excessive consumption, are clustered with the same criteria of the RE block. The questioning procedure directs the designer towards the RE block (line 19) if the costs concern the user of the system, whilst it guides towards the PR block (line 20) if the expenditures characterize the production process.

\subsubsection{The Logical Block Process (PR Block)}

This logical block concerns the investigation of further criticalities related to the manufacturing or delivering process. The user is directed towards the block when the formulated undesired effect, which hasn't allowed the identification of a contradiction, arises during the production process or characterizes the whole lifecycle of the system, as well as when unsatisfactory performances, flaws or high costs originate during the manufacturing stage. The scope of PR block is to reformulate the negative effect and the element that provokes it (line 21), downstream the individuation of the critical issues concerning the operations that concern with the production of the system. Since the focus of the investigation could be moved from the product to the design, manufacturing and assembling phases, the questions let the user change even the system to be analyzed (line 22).

\subsection{Implementation of the Algorithm in a Web Based Application}

In order to allow an extended testing activity, the algorithm has been implemented in a web platform. Thanks to this choice, each tester holds the possibility to extensively proof the dialogue based system and the analyzers can observe the whole set of results remotely.

The so built web application allows to reuse, in the formulation of downstream questions, the terms and concepts introduced by the users, thus providing a clearer explanation of the system's requests along the dialogue between the human and the computer. In the perspective of linking the TRIZ-based problem solving procedure 
with a knowledge search, as for example proposed in [9], a web application results the most convenient resource to speed up the information retrieval process.

\section{Testing Activity and Discussion}

The present section, first describes the organization of the testing campaign set up to validate the proposed algorithm. Then, the results of the experimental activity are discussed in terms of efficiency, estimating the effectiveness of the system through a comparison of the outputs with previous experiences and its robustness, by evaluating the repeatability of the outcomes.

\subsection{Test Group and Test Cases}

The proposed dialogue-based algorithm for problem solving has been tested by 30 Master Degree students in Mechanical Engineering at Università di Firenze (Florence, Italy) and at Politecnico di Milano (Milan, Italy). All these students had firstly received 20 lecture hours about TRIZ fundamentals; then, carried out manual tests have highlighted extremely different proficiency results in terms of their problem solving skills.

Further tests of the elaborated dialogue-based system have been performed by a group of $4 \mathrm{PhD}$ students and a postdoctoral research fellow in Mechanical Engineering with no TRIZ background, in order to appreciate differences and similarities according to different level of competences.

The testing activity has been carried out in computer laboratories where each person, in at most 90 minutes, had to analyze one of three real industrial problems, that were unknown for the participants, in order to obtain unbiased outcomes.

The problems were related to:

- A: Reducing energy waste from an anodizing tank, without creating obstacles to the process;

- B: Extreme wear of the fringe curtain in a X-ray inspection system for food industry;

- C: Complete transfer of oxygen from a large cylinder to several smaller ones, without any compressor.

These problems have been chosen for their different characteristics, in order to evaluate the different capabilities of the algorithm in driving the user towards the logical blocks of the dialogue-based system related with the task (removal of a negative effect, implementation of a new function, enhancement of a given performance), which was considered the most proper for each case study. Although each problem structure depends on the user interpretation [10], it is expected that people model the case A as a typical TRIZ contradiction or as a situation where a given performance is required. The case B clearly points to a negative effect, but should be preferentially modelled as a contradiction, while the case $\mathrm{C}$ should address the problem solver towards the implementation of a new performance or the improvement of an existing one. 
Finally, the three cases have been divided as follows:

- Case A: 11 students and 2 PhD students;

- Case B: 13 students and 1 PhD student;

- Case C: 6 students, $1 \mathrm{PhD}$ Student and 1 postdoctoral research fellow.

\subsection{Overview of the Results and Discussion}

The results of the problem situation analysis have been preliminarily evaluated by experts without any formal criterion, according to the following metrics:

- a good result is characterized by a precise description of the problem, without mistakes or misinterpretations, as well as by an appropriate set of features and elements required by the dialogue based system, viable to lead towards an appropriate information retrieval;

- a satisfactory result is characterized by a global comprehension of the problem under investigation, with an almost complete description of its main characteristics; the available information about the problem can support a preliminary direction of research for information gathering;

- an unsatisfactory result relates to a poor description of the problem, rich of misinterpretations and with no useful information capable to enlarge the potential solution space.

Table 2 provides an overall outlook of the results gathered by the Master Degree Students from both the Universities; PhD students were considered separately. In the assigned time, more than the $60 \%$ of the Master Degree Students were driven towards one of the final nodes of the algorithm, as well as 23 out of $30(76,6 \%)$ gave at least a satisfactory description of the problem situation. However, just a small part of them $(13,3 \%$ of the grand total) formulated a complete model of contradiction. A comparison between the Master Degree students from both the academic institution, does not highlight particular differences, since they obtained positive results for about the $75 \%$ of the analysis (approximately $80 \%$ in Florence and $70 \%$ in Milan). However, it is important to mention that students from Politecnico di Milano totally got better quality results (good, roughly 54\%; satisfactory, 18\%) than their mates from University of Florence (good, about 37\%; satisfactory 42\%).

Table 2. Overview of the results: quality of the problem description and percentage of formulated contradictions

\begin{tabular}{|c|c|c|c|}
\hline & $\begin{array}{c}\text { Florence } \\
\text { students }\end{array}$ & $\begin{array}{c}\text { Milan } \\
\text { students }\end{array}$ & $\begin{array}{c}\text { Students from both } \\
\text { Institutes }\end{array}$ \\
\hline Completed procedure & $63,2 \%$ & $63,6 \%$ & $63,3 \%$ \\
\hline Formulated contradiction & $10,5 \%$ & $18,2 \%$ & $13,3 \%$ \\
\hline Good result & $36,8 \%$ & $54,5 \%$ & $43,3 \%$ \\
\hline Satisfactory result & $42,1 \%$ & $18,2 \%$ & $33,3 \%$ \\
\hline Unsatisfactory result & $21,1 \%$ & $27,3 \%$ & $23,3 \%$ \\
\hline
\end{tabular}


The students who properly formulated a contradiction through the dialogue-based system achieved the best results in terms of abstraction according to the Hill Model: they got to the description of a physical contradiction and also identified the main characteristics that the solution should have in order to positively overcome the problem. Consistently with the problem solving models proposed in section 4.1 the algorithm has proved to be successful in stimulating the user in refining the problem under investigation, by asking him/her whether the detail level of the analysis is correct, allowing consequently to focus on different hierarchical levels of the system, thus moving upwards or downwards in the System Operator (more than $50 \%$ of the students have modified their initial definition of "system").

The convergent process described by the Funnel Model emerges by analyzing the body of results produced within this testing activity: the students, by following similar paths of investigation, frequently converged towards the same problem model, answering the questionnaire with similar definitions for the needed variables, even if, in many cases, this hasn't resulted sufficient for formulating an appropriate contradiction.

By thoroughly investigating the procedures carried out by the students that obtained good results, it's important to highlight that many of them achieved great benefits by changing the definition of the "technical system": they progressively changed the scope of the problem by identifying the right detail level and the critical features to be improved or to be removed. It is noticeable that all these students, regardless of the test case under analysis, considered the problem related to unsatisfactory or even missing performances of the technical system. The iteration of the procedure gave them a different perspective of the whole problem and by means of problem reformulation one third of them identified a critical contradiction for the problem solution. The students of this group that didn't get to the definition of a contradiction anyway leveraged their knowledge in order to give a description that made sense and however viable for a profitable information retrieval. The greatest part of these students (about 85\%) came indeed to one of the final nodes of the procedure with positive conclusions.

On the other hand, the students that didn't succeed in obtaining valuable results often followed an odd logic since they experienced some difficulties in distinguishing between elements/components of the system and their related parameters. About half of them tried to force the procedure towards the direction of a solution they had intuitively elaborated, rather than using the dialogue based system as a guiding tool to gradually explore the characteristics of the problem under investigation. Differently from their colleagues that obtained positive result, the greatest part (around 57\%) of these students didn't get to the end of the procedure, without taking therefore advantages from the refinement of the definition of the system.

It's equally important to verify whether the goal of approaching the problem with the right branch of the procedure has been met or not. By considering the sequence of steps that all the students went through, a simple analysis of Pearson's correlation [11] has been carried out, remarking that the students, regardless their success in exploiting the procedure, followed very similar paths of analysis. Indeed, the lower value of correlation between couples of exercises is approximately 0,72 ; such evidence can be considered from two different points of view. First, the individual cognitive processes are sensitive to similar education programs; second, the procedure 
doesn't have the expected capability of driving the user in the distinction of different issues. In order to remove this ambiguity, it is worth to carry out further tests with different people from various expertise fields and with different vocational experiences.

Table 3. Network of logical blocks and outputs of the questioning procedure

\begin{tabular}{|c|c|c|c|}
\hline & Anodizing tank & X rays & Cylinders \\
\hline Completed procedure & $72,7 \%$ & $66,7 \%$ & $53,8 \%$ \\
\hline Formulated contradiction & $9,1 \%$ & $16,7 \%$ & $15,4 \%$ \\
\hline Good result & $54,5 \%$ & $33,3 \%$ & $38,5 \%$ \\
\hline Satisfactory result & $45,5 \%$ & $0,0 \%$ & $38,5 \%$ \\
\hline Unsatisfactory result & $0,0 \%$ & $66,7 \%$ & $23,1 \%$ \\
\hline
\end{tabular}

Along the analysis of the results, it is also worth noting that the algorithm still presents some weak points, since the success of the test seems to depend upon the case study. In detail the three case studies led to significantly different overall results, as depicted in Table 3 .

The "Anodizing Tank" test case (A) produced the best results since no student produced any unsatisfactory description of the problem; on the contrary the case study about "X-rays detection system" (B) obtained very contradictory results: all the positive description are good, however the largest part (almost 70\%) are definitely unsatisfactory. The tests about "Cylinders" provided, as well, intermediate outcomes. The heterogeneous distribution of satisfactory results encourages to carry out further tests in order to better understand the adjustments that the algorithm requires.

Another lack emerged by analyzing the results, concerns the marginal capability of the procedure to point out features related to different time periods and situations, as in the logic of System Operator. Just 1 tester out of 30 has individuated problems and resources pertaining circumstances that are displayed both before and after the operational time of the main function of the investigated system. The majority of the students, more precisely 23 out of 30 , have identified resources useful for problem solving either before or after the reference operational time. On the other hand, 6 students have taken into account in their analysis just characteristics that are relevant within the time interval of the carried out primary function. The development of the algorithm has to take therefore into account strategies to investigate the resources and the characteristics of the technical system and its surroundings along the whole lifecycle of the analyzed object. The advantages of such more detailed survey have to be consequently examined in terms of improved capabilities to describe the problem situation and formalize contradictions.

For what concerns the potential differences in the solving path followed by more specialized people, the group formed by the $\mathrm{PhD}$ students and the postdoctoral research fellow produced only good or satisfactory results. In three cases they got to a good formulation of a contradiction, thus abstracting the problem and identifying the main features of the solution. In the remaining two cases the description of the problem is just satisfactory, but useful to perform a preliminary information search 
through the available sources (patents, scientific and technical literature), through which it is possible to enhance and refine the analysis itself or to find proper solutions.

\subsection{Comparing Computer-Aided and Individual Problem Solving Skills}

As clarified in Section 3.2 the same test group of MS students had been previously involved in manual tests without any computer support. The proof was carried out with the possibility to access their own books and the slides of the 20 hours course they had attended. Nevertheless, the amount of lecturing hours about TRIZ they were subjected is not deemed to particularly enhance individual problem solving skills.

By comparing the overall outcomes of the manual tests with traditional tools, giving indications about students' problem solving skills, and those obtained through the proposed dialogue based system, the share of students showing negative results drops from roughly $35 \%$ to about $27 \%$.

However, an in-depth analysis of the results highlights that students that had shown good problem solving skills (approximately $46 \%$ of the grand total) didn't obtain particular benefits in approaching the situation by means of the dialogue-based system (Table 4). One third of them described the problem by modelling it correctly, while the remaining two thirds produced a worse analysis than the one they could arrange without guided support (although with different case studies). It is worth to mention that just $15 \%$ of them produced a complete unsatisfactory model of the problem, while the others depicted it at least at a satisfactory level. On the contrary, the greatest benefits of the procedure (Table 4) emerge with those students that had previously showed more limited (just satisfactory or even poor) skills in the employment of systematic problem solving methods and tools (respectively $19 \%$ and $35 \%$ of the grand total). In fact, $80 \%$ of the first ones and $56 \%$ of the second ones described the problem in a more appropriate way than they had been capable without exploiting any support. The carried out analysis helped them in both correctly identifying spaces of solution that hadn't previously emerged because of psychological inertia or lack of knowledge and narrowing down the direction of research for useful information aimed at problem solving purposes.

Table 4. Positive and negative outcomes of the proposed system compared with the different Problem Solving skills of the students that carried out the testing activities

\begin{tabular}{|c|c|c|c|}
\hline & $\begin{array}{c}\text { Whole } \\
\text { test group } \\
\text { [\%] }\end{array}$ & $\begin{array}{c}\text { Positive outcomes } \\
\text { with the proposed } \\
\text { system [\%] }\end{array}$ & $\begin{array}{c}\text { Negative } \\
\text { outcomes with the } \\
\text { proposed system } \\
{[\%]}\end{array}$ \\
\hline $\begin{array}{c}\text { Students showing good } \\
\text { problem solving skills }\end{array}$ & $46,2 \%$ & $83,3 \%$ & $16,7 \%$ \\
\hline $\begin{array}{c}\text { Students showing } \\
\text { satisfactory problem solving } \\
\text { skills }\end{array}$ & $19,2 \%$ & $80,0 \%$ & $20,0 \%$ \\
\hline $\begin{array}{c}\text { Students showing poor } \\
\text { problem solving skills }\end{array}$ & $34,6 \%$ & $55,6 \%$ & $44,4 \%$ \\
\hline
\end{tabular}




\subsection{Proposals for the Enhancement of the Dialogue-Based System}

The limited capabilities shown by the algorithm in terms of exploring the topic of the investigation along the system lifecycle represents a primary issue for the improvement of the procedure. The capability to map the features related to the past and the future of the system represents a further opportunity for monitoring the mutual relations among the parameters and the elements that are relevant in the display of the undesired effects. With this perspective the authors are working on the introduction of a novel logical block, System Operator (labelled as SO), regarding the investigation of the thorough timeline during which suitable actions are viable to eliminate or attenuate the main undesired effects of the system. On the basis of the authors' vision, the user can be guided throughout such deeper investigation, whenever, with reference to the current version of the algorithm (lines 4-9), no successful formulation of a contradiction is emerging. The outputs of the block can be a hint to move the focus of the analysis towards a different system (thus linking to IS) or the individuation of control parameters relevant within a conflicting issue (thus addressing to AR). The last two options are represented in Fig. 2 by the lines 23 and 24 , respectively, while a novel flop in defining the contradiction is remarked by the line 25 .

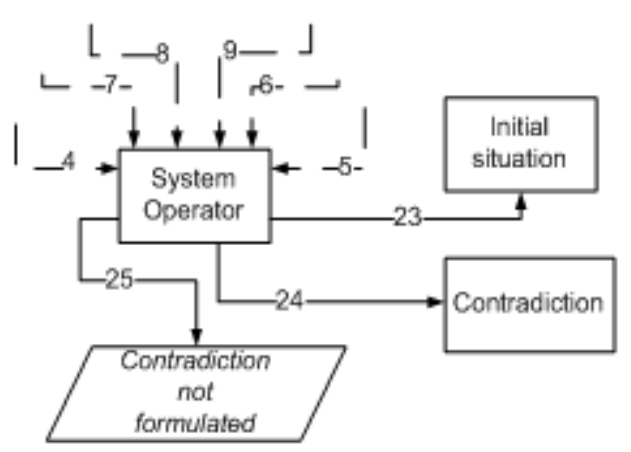

Fig. 2. Partial network of logical blocks regarding the modification of the current procedure

A further planned measure to be attained regards the introduction of graphical elements to depict, after each answer by the user, the current schematic representation of the problem described so far. According to the authors' thought the pictorial display of the topic schematization is viable to facilitate the learning process of the logic and to highlight further mistakes and/or misinterpretations concerning the introduced variables

\section{Conclusions and Future Activities}

The present paper proposes a model for computer-aided systematic problem solving, which has been adopted as a reference for the development of an original algorithm aimed at guiding designers, even without any TRIZ background, in the generation of 
inventive conceptual solutions. The tool has been built aiming at fulfilling the requirements that should characterize CAI problem solving systems, emerged in [3], and regarding the problem investigation tasks. Such requirements refer to the capability to face the distinctive aspects of design problems and to perform a detailed description of the initial situation, beyond the advantages originated by the employment of natural language within dialogue-based systems.

The algorithm has been implemented in a prototype web application already tested with $\mathrm{MS}$ and $\mathrm{PhD}$ students both from Politecnico di Milano and University of Florence, obtaining positive results especially with the students with poorer systematic problem solving skills.

The tests performed so far have demonstrated that the proposed system is suitable to combine several expected benefits of the most acknowledged problem solving techniques. First, cognitive capabilities are enhanced by soliciting the analysis of the problem from different perspectives, thus overcoming psychological inertia as typically addressed by brainstorming sessions and the TRIZ System Operator. Compared with brainstorming, the analysis follows a more systematic path, avoiding useless trial \& errors, with consequent benefits in terms of process efficiency. The personal knowledge of the user is leveraged to solve the analyzed problem by suggesting suitable TRIZ separation principles, once that a proper model of contradiction limiting the applicability of typical solutions has been built. Indeed, while the overall results of the test have been satisfactory, the proposed algorithm needs to be definitely improved in the support to the identification of a proper model of contradiction, mostly in terms of recognizing the critical design parameter characterized by conflicting requirements in order to satisfy different evaluation parameters.

The system is also structured in order to elicit lacks of knowledge by the user, either in terms of limited understanding of the mechanism originating the problem, or missing physical/chemical effects suitable to deliver a certain function. Such knowledge lacks will be used as inputs for a patent-mining tool capable to extract relevant information from patent texts within or even outside the problem domain. The complete system will be tested within a project of the EraSME EU Programme, by involving Small and Medium Enterprises from Italy and Spain.

\section{Acknowledgments}

This research is partially funded by the EraSME EU Programme. The authors would like to thank drWolf srl, coordinator of the project "IT Tool to support SMEs in systematic innovation based on open innovation paradigm" for the inputs provided to the development of the present activity. Special thanks are also dedicated to Nikholai Khomenko for the valuable suggestions at the beginning of this research.

\section{References}

1. Cascini, G., Jantschgi, J., Khomenko, N., Murashkovska, I., Sokol, A., Tomasi, F.: TETRIS: Teaching TRIZ at School - Meeting the educational requirements of heterogeneous curricula. In: Vaneker, T., Lutters, E. (eds.) Proceedings of the 8th ETRIA TRIZ Future Conference, Enschede, The Netherlands, November 5-7, pp. 123-130 (2008) 
2. Dubois, S., Lutz, P., Rousselot, F.: Proposal of an object-oriented model of the physical contradiction to facilitate the problem-framing phase in design. In: Proceedings of the 3th ETRIA TRIZ Future Conference, Aachen, Germany, November 12-14 (2003)

3. Becattini, N., Borgianni, Y., Cascini, G., Rotini, F.: Computer-Aided Problem Solving Part 2: Objectives, Approaches, Identification of System Requirements. In: Cavallucci, D., De Guio, R., Cascini, G. (eds.) CAI 2011. IFIP AICT, vol. 355, pp. 117-131. Springer, Heidelberg (2011)

4. Cavallucci, D., Khomenko, N.: From TRIZ to OTSM-TRIZ: addressing complexity challenges in inventive design. Int. J. Prod. Dev. 4, 4-21 (2007)

5. Adunka R.: Lessons Learned in the Introduction of TRIZ at Siemens A\&D. In: Proceedings of the 7th ETRIA TRIZ Future Conference, Frankfurt, Germany, November 6-8 (2007)

6. Lee, J.Y.: Implementing TRIZ at Samsung Electronics. In: Proceedings of the Global TRIZ Conference in Korea (2010)

7. Kohn, S., Hüsig, S.: Potential benefits, current supply, utilization and barriers to adoption: An exploratory study on German SMEs and innovation software. Technovation 26, 988-998 (2006)

8. Vermaas, P.E.: The Flexible Meaning of Function in Engineering. In: Norell Bergendahl, M., Grimheden, M., Leifer, L., Skogstad, P., Lindemann, U. (eds.) Proceedings of the 17th International Conference on Engineering Design (ICED 2009), Stanford, vol. 2, pp. 113-124 (2009)

9. Cascini, G., Russo, D.: Computer-Aided analysis of patents and search for TRIZ contradictions. Int. J. Prod. Dev., Special Issue: Creativity and Innovation Employing TRIZ 4, 52-67 (2007)

10. Simon, H.A.: The structure of ill-structured problems. Artif. Intell. 4, 181-201 (1973)

11. National Institute of Standards and Technology (NIST), e-Handbook of Statistical Methods (2003), obtained from the web http://www.itl.nist.gov/div898/handbook 